\title{
The comparison of clinical outcomes of endophthalmitis from fluoroquinolone- resistant and susceptible bacteria
}

This article was published in the following Dove Press journal:

Clinical Ophthalmology

26 March 2010

Number of times this article has been viewed

\author{
Anita R Shirodkar \\ Harry W Flynn Jr \\ Kyle Alliman \\ Geeta A Lalwani \\ Chrisfouad Alabiad \\ Andrew A Moshfeghi \\ Darlene Miller \\ Department of Ophthalmology, \\ Bascom Palmer Eye Institute, \\ University of Miami Miller School \\ of Medicine, Miami, FL, USA
}

Correspondence: Anita R Shirodkar Bascom Palmer Eye Institute, 900 NW 17th Street, Miami, FL 33136, USA

Tel +l 3053266118

Fax + I 3053266417

Email ashirodkar@med.miami.edu
Purpose: To identify patients who developed acute-onset endophthalmitis after clear corneal cataract surgery, and to compare treatment outcomes between cases caused by fluoroquinolone susceptible organisms versus fluoroquinolone resistant organisms.

Design: Retrospective case series.

Methods: Patients who developed endophthalmitis within six weeks of cataract surgery, and were treated between January 1996 and December 2008 at Bascom Palmer Eye Institute in Miami, Florida, were identified retrospectively. Clinical features, organisms cultured, and visual acuity outcomes were evaluated.

Results: A total of 97 patients met study criteria, and 37 (38\%) demonstrated in vitro fluoroquinolone resistance. All fluoroquinolone resistant endophthalmitis in the study was caused by either Staphylococcus epidermidis $(\mathrm{n}=32)$ or Staphylococcus aureus $(\mathrm{n}=5)$. Presenting clinical features were similar between fluoroquinolone resistant and fluoroquinolone susceptible groups. Final visual acuity was $\geq 20 / 40$ in $49 \%$ of fluoroquinolone-resistant cases and $42 \%$ of fluoroquinolonesusceptible cases. All fluoroquinolone-resistant isolates were susceptible to vancomycin.

Conclusion: In the current study, approximately one-third of isolates were resistant to fluoroquinolones. There was no significant difference in clinical outcomes in this study, regardless of fluoroquinolone susceptibility.

Keywords: endophthalmitis, fluoroquinolones

\section{Introduction}

Endophthalmitis is a serious but uncommon complication following cataract surgery. The incidence of endophthalmitis after cataract surgery is reported to be between $0.05 \%$ and $0.68 \%{ }^{1-5}$ Topical fluoroquinolone (FQ) antibiotics are commonly used for prophylaxis, during the perioperative period for cataract surgery. Prophylactic use of topical FQs may theoretically reduce the rate of endophthalmitis by lowering the bacterial load on the ocular surface.$^{6-8}$ However, the actual effectiveness of using FQs to prevent acute-onset endophthalmitis after cataract surgery remains controversial, particularly since endophthalmitis may still occur after the use of antibiotic prophylaxis. ${ }^{9}$ Although prophylactic antibiotic use is often left to the discretion of the cataract surgeon, preoperative use of povidone-iodine has become the standard of care. ${ }^{10-12}$ This 12-year study reports on treatment outcomes in acute-onset endophthalmitis after clear corneal cataract surgery and compares outcomes between FQ-resistant and FQ-susceptible organisms.

\section{Methods}

Microbiology records were used to identify all cases of culture-positive endophthalmitis at Bascom Palmer Eye Institute in Miami, Florida from January 1996 to December 
2008. Clinical records were retrospectively accessed of these identified cases, and all patients with endophthalmitis within six weeks of clear corneal cataract surgery were included in the study. The study included patients who underwent surgery at an outside institution and were referred for care as well as patients who underwent surgery at Bascom Palmer Eye Institute. Patients excluded were those who underwent nonclear corneal cataract surgery, had combined ophthalmic surgical procedures, and patients who developed endophthalmitis greater than six weeks after cataract surgery. All patients received initial treatment with intravitreal antibiotics. A vitreous specimen was also taken at diagnosis. The decision to perform a vitrectomy was made by the treating ophthalmic physician, generally in accordance with the evidence from the Endophthalmitis Vitrectomy Study. ${ }^{3}$ Recorded data included clinical features, visual acuity at diagnosis, cultured organisms and antibiotic susceptibilities, and visual acuity at followup. FQ resistance in an organism was defined by the lack of susceptibility to ciprofloxacin, levofloxacin, moxifloxacin, or gatifloxacin.

\section{Results}

In the 12-year time period, 97 patients met study criteria. Of these 97 patients, 37 (38\%) had endophthalmitis caused by FQ-resistant organisms and 60 (62\%) had endophthalmitis caused by FQ-susceptible organisms. These groups had similar presenting clinical features (Table 1). Of the FQ-resistant group, 35/37 (95\%) had a presenting visual acuity of $<5 / 200$, whereas $48 / 60(80 \%)$ had a presenting visual acuity of $<5 / 200$ in the FQ-susceptible group. Hypopyon was present in $32 / 37$ (86\%) and 50/60 (83\%) of the FQ-resistant and -susceptible groups, respectively. On average, the diagnosis of endophthalmitis was made at postoperative day 12 after cataract surgery in the FQ-resistant group and at postoperative day 13 in the FQ-susceptible group.

Visual acuities at final followup were similar between the FQ-resistant and -susceptible groups (Table 2). Final visual acuity $\geq 20 / 100$ was achieved in 27/37 (73\%) of patients in the FQ-resistant group, and 36/60 (60\%) of patients in the FQ-susceptible group. Final visual acuity of no light

Table I Presenting clinical characteristics of patients with endophthalmitis following clear corneal cataract surgery

\begin{tabular}{llll}
\hline Total $\mathbf{n}=\mathbf{9 7}$ & VA $<\mathbf{5 / 2 0 0}$ & Hypopyon & Days to diagnosis \\
\hline FQ resistant & 35 & 32 & 12 \\
$(\mathrm{n}=37)$ & $(95 \%)$ & $(86 \%)$ & \\
FQ susceptible & 48 & 50 & 13 \\
$(\mathrm{n}=60)$ & $(80 \%)$ & $(83 \%)$ & \\
\hline
\end{tabular}

Table 2 Final visual acuities by fluoroquinolone susceptibility

\begin{tabular}{lllll}
\hline Total $\mathbf{n}=\mathbf{9 7}$ & $\geq \mathbf{2 0 / 4 0}$ & $\geq \mathbf{2 0 / 1 0 0}$ & $<\mathbf{5 / 2 0 0}$ & $\mathbf{N L P}$ \\
\hline FQ resistant & 18 & 27 & 9 & 2 \\
$(\mathrm{n}=37)$ & $(49 \%)$ & $(73 \%)$ & $(24 \%)$ & $(5 \%)$ \\
FQ susceptible & 25 & 36 & 15 & 4 \\
$(\mathrm{n}=60)$ & $(42 \%)$ & $(60 \%)$ & $(25 \%)$ & $(7 \%)$ \\
\hline
\end{tabular}

perception (NLP) was noted in 2/37 (5\%) and 4/60 (7\%) of patients in the FQ-resistant and FQ-susceptible groups, respectively.

The endophthalmitis cases were also classified by causative organism in both the FQ-susceptible and FQ-resistant groups (Tables 3 and 4). Of the 60 FQ-susceptible cases, 27 (45\%) were caused by Staphylcoccus epidermidis, four were caused by S. aureus (7\%), and 29 (48\%) were caused by other organisms, including Streptococcus and Gram-negative species. Amongst the FQ-susceptible S. epidermidis, 23/27 (85\%) had a final visual acuity of $\geq 20 / 100$ and no patients had a final visual acuity of NLP. Of the FQ-susceptible $S$. aureus, $1 / 4$ (25\%) had a final visual acuity of $\geq 20 / 100$ and also no patients had a final visual acuity of NLP. Regarding the other causative organisms in the FQ-susceptible group, 12/29 (41\%) had a final visual acuity of $\geq 20 / 100$ and $4 / 29$ (14\%) had a final visual acuity of NLP.

Of the 37 FQ-resistant cases, 32 (86\%) were caused by $S$. epidermidis, and five (14\%) were caused by $S$. aureus. Amongst the FQ-resistant S. epidermidis, 25/32 (78\%) had a final visual acuity of $\geq 20 / 100$ and no patients had a final visual acuity of NLP. In the FQ-resistant $S$. aureus group, $2 / 5(40 \%)$ patients had a final visual acuity of $\geq 20 / 100$ and $2 / 5(40 \%)$ patients had a final visual acuity of NLP. Of the FQ-resistant $S$. aureus group, $3 / 5$ (60\%) were also methicillin-resistant; two of these patients had a final visual acuity of NLP, and the remaining one had a final visual acuity of 20/100. The two patients in the FQ-resistant S. aureus group with methicillin-susceptible organisms had final visual acuities of 20/40 and HM.

Table 3 Final visual acuities by cultured organism in fluoroquinolone susceptible cases

\begin{tabular}{lllll}
\hline Total $\mathbf{n}=\mathbf{6 0}$ & $\geq \mathbf{2 0 / 4 0}$ & $\geq \mathbf{2 0 / 1 0 0}$ & $<\mathbf{5 / 2 0 0}$ & NLP \\
\hline $\begin{array}{l}\text { S. epidermidis } \\
(\mathrm{n}=27)\end{array}$ & 17 & 23 & $\mathrm{I}$ & 0 \\
S. aureus & $(63 \%)$ & $(85 \%)$ & $(4 \%)$ & \\
$(\mathrm{n}=4)$ & $\mathrm{I}$ & $\mathrm{I}$ & 3 & 0 \\
$\begin{array}{l}\text { Other } \\
(\mathrm{n}=29)\end{array}$ & $(25 \%)$ & $(25 \%)$ & $(75 \%)$ & \\
\hline
\end{tabular}

a Other cultured organisms included Streptococcus and gram-negative species. 
Table 4 Final visual acuities by cultured organism in fluoroquinolone resistant cases

\begin{tabular}{lllll}
\hline Total $\mathbf{n}=\mathbf{3 7}$ & $\geq \mathbf{2 0 / 4 0}$ & $\geq \mathbf{2 0 / 1 0 0}$ & $<\mathbf{5 / 2 0 0}$ & NLP \\
\hline $\begin{array}{l}\text { S. epidermidis } \\
(\mathrm{n}=32)\end{array}$ & 17 & 25 & 4 & 0 \\
S. aureus & $(53 \%)$ & $(78 \%)$ & $(13 \%)$ & \\
$(\mathrm{n}=5)$ & $\mathrm{I}$ & 2 & $\mathrm{I}$ & 2 \\
\hline
\end{tabular}

Organisms resistant to FQs were 100\% susceptible to vancomycin. These FQ-resistant organisms were also noted to be $66 \%$ susceptible to gentamicin, trimethoprim/ sulfamethoxazole, and clindamycin.

\section{Discussion}

Endophthalmitis can be a devastating complication after cataract surgery. In an attempt to reduce the rates of this complication, topical fluoroquinolones are commonly used as prophylaxis in the perioperative period of cataract surgery at the discretion of the operating surgeon. The topical use of FQs theoretically decreases the viable organisms that enter the eye intraoperatively. FQs may achieve high intraocular concentrations via corneal penetration of the drug, but their prophylactic use is controversial. ${ }^{11}$ Some studies have suggested that use of topical perioperative FQs does not decrease the incidence of culture-positive endophthalmitis after cataract surgery. ${ }^{9,13}$ The use of intracameral antibiotics has also been recommended as an effective method of prophylaxis. ${ }^{14-16}$ The European Society of Cataract and Refractive Surgeons (ESCRS) Endophthalmitis Study Group reported a 4.92-fold increase in the risk for total postoperative endophthalmitis in their study of 16,603 patients, $82 \%$ of which underwent clear corneal cataract surgery. ${ }^{17}$ A total of 29 patients $(0.17 \%)$ presented with endophthalmitis in that study, which is higher than the $0.03 \%$ incidence previously reported at our institution. ${ }^{1,17}$

Table 5 Organisms and clinical features of culture-proven, acuteonset endophthalmitis: comparison of EVS, ESCRS, and current studies $3,14,17-19$

\begin{tabular}{|c|c|c|c|}
\hline & EVS & ESCRS & Current study \\
\hline Number of patients & 291 & 19 & 97 \\
\hline$\%$ coagulase $(-)$ & $70 \%$ & $42 \%$ & $62 \%$ \\
\hline \multicolumn{4}{|l|}{ Staphylococcus ${ }^{\mathrm{a}}$} \\
\hline$\%$ S. aureus & $10 \%$ & $11 \%$ & $9 \%$ \\
\hline$\%$ other organisms ${ }^{b}$ & $20 \%$ & $53 \%$ & $29 \%$ \\
\hline$\% \vee A \geq 20 / 40$ & $52 \%$ & $53 \%$ & $44 \%$ \\
\hline
\end{tabular}

alncludes S. epidermidis species.

Includes Streptococcus and gram (-) species.
The findings of the current study were comparable to those of the ESCRS study and the Endophthalmitis Vitrectomy Study (EVS) for cases of culture-proven, acute-onset endophthalmitis (Table 5). The organisms cultured and the percentage of patients with a final visual acuity of $\geq 20 / 40$ was similar among the three studies. ${ }^{18,19}$

Also of note, the treatment protocol in the EVS included intravitreal injections of vancomycin and amikacin and subconjunctival injections of vancomycin, ceftazidime, and dexamethasone. ${ }^{3}$ In the current study, all of the patients with endophthalmitis received treatment consisting of only intravitreal injections of vancomycin, ceftazidime, and dexamethasone. As previously reported, no difference was noted in clinical outcomes after acute-onset endophthalmitis using the latter intravitreal regimen. ${ }^{1}$

Limitations to this study include its retrospective nature; since most of the study patients were referred from outside institutions, data on prophylactic preoperative or postoperative use of antibiotics were not available. Additionally, data related to preoperative vision and potential intraoperative surgical complications were unavailable. Another limitation is that it remains unclear whether the endophthalmitis in FQ-resistant cases was caused by initially FQ-resistant organisms or if the FQ resistance was developed by an initially FQ-susceptible organism during the course of the infection. FQs remain an important antibiotic, but coverage of Staphylococcus species remains inconsistent. ${ }^{20}$ All FQ-resistant organisms in this study were susceptible to vancomycin, and a significant proportion of the organisms were also susceptible to tetracyclines, gentamicin, trimethoprim/sulfamethoxazole, and clindamycin.

In the current study, approximately one-third of isolates cultured from acute-onset endophthalmitis after cataract surgery were resistant to FQs. Both the FQ-resistant and FQ-susceptible groups had similar presenting clinical features, and the decision for immediate vitrectomy versus intravitreal tap and inject was made with the suggestions of the EVS. ${ }^{3}$ No significant difference in clinical outcomes was noted, regardless of FQ susceptibility.

\section{Disclosures}

This work was funded in part by The Palm Beach Community Trust Fund, Palm Beach, FL, USA; An unrestricted grant from Research to Prevent Blindness Inc., New York, NY, USA; and the National Institutes of Health NEI Center Grant P30 EY014801. The study protocol was approved by the Institutional Review Board of the University of Miami Miller School of Medicine in Miami, Florida. 


\section{References}

1. Lalwani GA, Flynn HW Jr, Scott IU, et al. Acute-onset endophthalmitis after clear corneal cataract surgery (1996-2005). Clinical features, causative organisms, and visual acuity outcomes. Ophthalmology. 2008;115(3):473-476.

2. Colleaux KM, Hamilton WK. Effect of prophylactic antibiotics and incision type on the incidence of endophthalmitis after cataract surgery. Can J Ophthalmol. 2000;35(7):373-378.

3. Endophthalmitis Vitrectomy Study Group. Results of the Endophthalmitis Vitrectomy Study. A randomized trial of immediate vitrectomy and of intravenous antibiotics for the treatment of postoperative bacterial endophthalmitis. Arch Ophthalmol. 1995;113(12):1479-1496.

4. Hammoudi DS, Abdolell M, Wong DT. Patterns of perioperative prophylaxis for cataract surgery in Canada. Can J Ophthalmol. 2007;42(5):681-688.

5. Jensen MK, Fiscella RG, Moshirfar M, et al. Third- and fourth-generation fluoroquinolones: retrospective comparison of endophthalmitis after cataract surgery performed over 10 years. J Cataract Refract Surg. 2008;34(9):1460-1467.

6. Miño de Kaspar H, Kreutzer TC, Aguirre-Romo I, et al. A prospective randomized study to determine the efficacy of preoperative topical levofloxacin in reducing conjunctival bacterial flora. Am J Ophthalmol. 2008;145(1):136-142.

7. Hori Y, Nakazawa T, Maeda N, et al. Susceptibility comparisons of normal preoperative conjunctival bacteria to fluoroquinolones. J Cataract Refract Surg. 2009;35(3):475-479.

8. Kowalski RP, Romanowski EG, Mah FS, et al. Topical prophylaxis with moxifloxacin prevents endophthalmitis in a rabbit model. Am JOphthalmol. 2004;138(1):33-37.

9. Deramo VA, Lai JC, Fastenberg DM, et al. Acute endophthalmitis in eyes treated prophylactically with gatifloxacin and moxifloxacin. Am J Ophthalmol. 2006;142(5):721-725.

10. Gordon-Bennett P, Karas A, Flanagan D, et al. A survey of measures used for the prevention of postoperative endophthalmitis after cataract surgery in the United Kingdom. Eye. 2008;22(5):620-627.
11. Ciulla TA, Starr MB, Masket S. Bacterial endophthalmitis prophylaxis for cataract surgery: an evidence-based update. Ophthalmology. 2002;109(1):13-24.

12. Thoms SS, Musch DC, Soong HK. Postoperative endophthalmitis associated with sutured versus unsutured clear corneal cataract incisions. Br J Ophthalmol. 2007;91(6):728-730.

13. Bohigian GM. A retrospective study of the incidence of culture-positive endophthalmitis after cataract surgery and the use of preoperative antibiotics. Ophthalmic Surg Lasers Imaging. 2007;38(2):103-106.

14. Endophthalmitis Study Group, European Society of Cataract and Refractive Surgeons. Prophylaxis of postoperative endophthalmitis following cataract surgery: results of the ESCRS multicenter study and identification of risk factors. J Cataract Refract Surg. 2007;33(6):978-988.

15. Barry P, Gardner S, Seal D, et al. Clinical observations associated with proven and unproven cases in the ESCRS study of prophylaxis of postoperative endophthalmitis after cataract surgery. $J$ Cataract Refract Surg. 2009;35(9):1523-1531.

16. Liesegang TJ. Intracameral antibiotics: questions for the United States based on prospective studies. J Cataract Refract Surg. 2008;34(3): 505-509.

17. Barry P, Gardner S, Seal D, et al; European Society of Cataract and Refractive Surgeons Endophthalmitis Study Group. Clinical observations associated with proven and unproven cases in the ESCRS study of prophylaxis of postoperative endophthalmitis after cataract surgery. J Cataract Refract Surg. 2009;35(9):1523-1531.

18. Endophthalmitis Vitrectomy Study Group. Microbiologic factors and visual outcome in the Endophthalmitis Vitrectomy Study. Am J Ophthalmol. 1996;122(6):830-846.

19. Han DP, Wisniewski SR, Wilson LA, et al. Spectrum and susceptibilities of microbiologic isolates in the Endophthalmitis Vitrectomy Study. Am J Ophthalmol. 1996;122(1):1-17.

20. Miller DM, Vedula AS, Flynn HW Jr, et al. Endophthalmitis caused by Staphylococcus epidermidis: in vitro antibiotic susceptibilities and clinical outcomes. Ophthalmic Surg Lasers Imaging. 2007;38(6):446-451.
Clinical Ophthalmology

\section{Publish your work in this journal}

Clinical Ophthalmology is an international, peer-reviewed journal covering all subspecialties within ophthalmology. Key topics include: Optometry; Visual science; Pharmacology and drug therapy in eye diseases; Basic Sciences; Primary and Secondary eye care; Patient Safety and Quality of Care Improvements. This journal is indexed on Submit your manuscript here: http://www.dovepress.com/clinical-ophthalmology-journal

\section{Dovepress}

PubMed Central and CAS, and is the official journal of The Society of Clinical Ophthalmology (SCO). The manuscript management system is completely online and includes a very quick and fair peer-review system, which is all easy to use. Visit http://www.dovepress.com/ testimonials.php to read real quotes from published authors. 